\title{
Existence of unbounded positive solutions for BVPs of singular fractional differential equations
}

Yuji Liua, Haiping Shib

${ }^{a}$ Department of Mathematics, Guangdong University of Business Studies, Guangzhou 510320, P.R.China.

${ }^{b}$ Basic Courses Department, Guangdong Construction Vocational Technology Institute, Guangzhou 510450, P.R.China.

This paper is dedicated to Professor Ljubomir Ćirić

Communicated by Professor V. Berinde

\section{Abstract}

In this article, we establish the existence of multiple unbounded positive solutions to the boundary value problem of the nonlinear singular fractional differential equation

$$
\left\{\begin{array}{l}
D_{0^{+}}^{\alpha} u(t)+f(t, u(t))=0, t \in(0,1), 1<\alpha<2 \\
{\left.\left[I_{0^{+}}^{2-\alpha} u(t)\right]^{\prime}\right|_{t=0}=0} \\
u(1)=0
\end{array}\right.
$$

Our analysis relies on the well known fixed point theorems in the cones in Banach spaces. Here $f$ is singular at $t=0$ and $t=1$. (C)2012. All rights reserved.

Keywords: Singular fractional differential equation, boundary value problem, unbounded positive solution, Fixed Point Theorem.

2010 MSC: Primary 34B37; Secondary 65L05; 92D25.

\section{Introduction}

Fractional differential equations have many applications in modeling of physical and chemical processes and in engineering and have been of great interest recently. In its turn, mathematical aspects of studies on fractional differential equations were discussed by many authors, see the text books $[1,2]$ and papers [3-10] and the references therein.

\footnotetext{
*Corresponding author

Email addresses: liuyuji888@sohu.com (Yuji Liu), haipingshi@sohu.com (Haiping Shi)
} 
The use of cone theoretic techniques in the study of solutions to boundary value problems has a rich and diverse history. In [3], the authors studied the existence of positive solutions (continuous and nonnegative on $[0,1]$ ) of the following boundary value problem for fractional differential equation

$$
\left\{\begin{array}{l}
D_{0^{+}}^{\alpha} x(t)+f(t, x(t))=0, t \in(0,1), 1<\alpha<2 \\
x(0)=x(1)=0
\end{array}\right.
$$

where $f:[0,1] \times[0, \infty) \rightarrow[0, \infty)$ is a continuous function, $D_{0^{+}}^{\alpha}\left(D^{\alpha}\right.$ for short $)$ is the Riemann-Liouville fractional derivative of order $\alpha$.

One notes that the problem

$$
u^{\prime \prime}(t)+1=0, \quad u^{\prime}(0)=u(1)=0
$$

has bounded solution, however, the generalized boundary value problem for fractional differential equation

$$
\left\{\begin{array}{l}
D_{0^{+}}^{\alpha} u(t)+1=0, t \in(0,1), 1<\alpha<2 \\
{\left.\left[I_{0^{+}}^{2-\alpha} u(t)\right]^{\prime}\right|_{t=0}=0} \\
u(1)=0
\end{array}\right.
$$

has solution

$$
u(t)=-\int_{0}^{t} \frac{(t-s)^{\alpha-1}}{\Gamma(\alpha)} d s+t^{\alpha-2} \int_{0}^{1} \frac{(1-s)^{\alpha-1}}{\Gamma(\alpha)} d s
$$

which is unbounded.

In papers $[6,7,9,10]$, the existence of positive solutions (continuous and nonnegative on [0,1]) of boundary value problems for fractional differential equations were studied, but there exists no paper concerned with the existence of unbounded positive solutions of boundary value problems for fractional differential equations.

Motivated by this reason, in this paper, we discuss the existence of multiple unbounded positive solutions to the boundary value problem of the nonlinear fractional differential equation of the form

$$
\left\{\begin{array}{l}
D_{0^{+}}^{\alpha} u(t)+f(t, u(t))=0, t \in(0,1), 1<\alpha<2 \\
{\left.\left[I_{0^{+}}^{2-\alpha} u(t)\right]^{\prime}\right|_{t=0}=0} \\
u(1)=0
\end{array}\right.
$$

where $D_{0^{+}}^{\alpha}$ ( $D^{\alpha}$ for short) is the Riemann-Liouville fractional derivative of order $\alpha$, and $f:(0,1) \times[0, \infty) \rightarrow$ $[0, \infty)$ such that $f\left(t, t^{\alpha-2} x\right)$ is continuous on $(0,1) \times[0, \infty) . f$ is singular at $t=0$ and $t=1$.

We obtain the existence results for two and three positive solutions of $\operatorname{BVP}(1.1)$ by using the fixed point theorems in the cones in Banach spaces.

\section{Preliminary results}

For the convenience of the reader, we present here the necessary definitions from fixed point theory and fractional calculus theory. These definitions and results can be found in the literatures [11].

Definition 2.1. Let $X$ be a real Banach space. The nonempty convex closed subset $P$ of $X$ is called a cone in $X$ if $a x \in P$ for all $x \in P$ and $a \geq 0, x \in X$ and $-x \in X$ imply $x=0$.

Definition 2.2. A map $\psi: P \rightarrow[0,+\infty)$ is a nonnegative continuous concave or convex functional map provided $\psi$ is nonnegative, continuous and satisfies

$$
\psi(t x+(1-t) y) \geq t \psi(x)+(1-t) \psi(y)
$$

or

$$
\psi(t x+(1-t) y) \leq t \psi(x)+(1-t) \psi(y)
$$

for all $x, y \in P$ and $t \in[0,1]$. 
Definition 2.3. An operator $T: X \rightarrow X$ is completely continuous if it is continuous and maps bounded sets into pre-compact sets.

Let $\psi$ be a nonnegative functional on a cone $P$ of a real Banach space $X$. Define the sets by

$$
\begin{aligned}
& P_{r}=\{y \in P: \quad\|y\|<r\} \\
& P(\psi ; a, b)=\{y \in P: \quad a \leq \psi(y),\|y\|<b\}, \\
& P(\psi, d):=\{x \in P: \psi(x)<d\} .
\end{aligned}
$$

Lemma 2.1. Let $T: \bar{P}_{c} \rightarrow \bar{P}_{c}$ be a completely continuous operator and let $\psi$ be a nonnegative continuous concave functional on $P$ such that $\psi(y) \leq\|y\|$ for all $y \in \bar{P}_{c}$. Suppose that there exist $0<a<b<d \leq c$ such that

(E1) $\{y \in P(\psi ; b, d) \mid \psi(y)>b\} \neq \emptyset$ and $\psi(T y)>b$ for $y \in P(\psi ; b, d)$;

(E2) $\|T y\|<a$ for $\|y\| \leq a$;

(E3) $\psi(T y)>b$ for $y \in P(\psi ; b, c)$ with $\|T y\|>d$.

Then $T$ has at least three fixed points $y_{1}, y_{2}$ and $y_{3}$ such that $\left\|y_{1}\right\|<a, b<\psi\left(y_{2}\right)$ and $\left\|y_{3}\right\|>a$ with $\psi\left(y_{3}\right)<b$.

Lemma 2.2. Suppose $P$ is a cone in a real Banach space $X, \alpha, \gamma: P \rightarrow I_{0}$ be two continuous increasing functionals, $\theta: P \rightarrow I_{0}$ be a continuous functional and there exist positive numbers $M, c>0$ such that

(i) $T: \bar{P}(\gamma, c) \rightarrow P$ is a completely continuous operator;

(ii) $\theta(0)=0$ and $\gamma(x) \leq \theta(x) \leq \alpha(x),\|x\| \leq M \gamma(x)$ for all $x \in \bar{P}(\gamma, c)$;

(iii) there exist constants $0<a<b<c$ such that $\theta(\lambda x) \leq \lambda \theta(x)$ for all $\lambda \in[0,1]$ and $x \in \partial P(\theta, b)$;

(iv) $\gamma(T x)>c$ for all $x \in \partial P(\gamma, c) ; \theta(T x)<b$ for all $x \in \partial P(\theta, b) ; P(\alpha, a) \neq \emptyset$ and $\alpha(T x)>a$ for all $x \in \partial P(\alpha, a)$;

then $T$ has two fixed points $x_{1}, x_{2}$ in $P(\gamma, c)$ such that

$$
\alpha\left(x_{1}\right)>a, \theta\left(x_{1}\right)<b<\theta\left(x_{2}\right), \quad \gamma\left(x_{2}<c .\right.
$$

Lemma 2.3. Suppose $P$ is a cone in a real Banach space $X, \alpha, \gamma: P \rightarrow I_{0}$ be two continuous increasing functionals, $\theta: P \rightarrow I_{0}$ be a continuous functional and there exist positive numbers $M, c>0$ such that (i), (ii) and (iii) in Lemma 2.4 hold and

(iv) $\gamma(T x)<c$ for all $x \in \partial P(\gamma, c) ; \theta(T x)>b$ for all $x \in \partial P(\theta, b) ; P(\alpha, a) \neq \emptyset$ and $\alpha(T x)<a$ for all $x \in \partial P(\alpha, a)$;

then $T$ has two fixed points $x_{1}, x_{2}$ in $P(\gamma, c)$ such that

$$
\alpha\left(x_{1}\right)>a, \theta\left(x_{1}\right)<b<\theta\left(x_{2}\right), \quad \gamma\left(x_{2}<c .\right.
$$

Definition 2.4. The Riemann-Liouville fractional integral of order $\alpha>0$ of a function $f:(0, \infty) \rightarrow R$ is given by

$$
I_{0+}^{\alpha} f(t)=\frac{1}{\Gamma(\alpha)} \int_{0}^{t}(t-s)^{\alpha-1} f(s) d s,
$$

provided that the right-hand side exists.

Definition 2.5. The Riemann-Liouville fractional derivative of order $\alpha>0$ of a continuous function $f:(0, \infty) \rightarrow R$ is given by

$$
D_{0^{+}}^{\alpha} f(t)=\frac{1}{\Gamma(n-\alpha)} \frac{d^{n+1}}{d t^{n+1}} \int_{0}^{t} \frac{f(s)}{(t-s)^{\alpha-n+1}} d s,
$$

where $n-1<\alpha \leq n$, provided that the right-hand side is point-wise defined on $(0, \infty)$.

Lemma 2.4. Let $n-1 \leq \alpha<n, u \in C^{0}(0,1) \cap L^{1}(0,1)$. Then

$$
I_{0+}^{\alpha} D_{0+}^{\alpha} u(t)=u(t)+C_{1} t^{\alpha-1}+C_{2} t^{\alpha-2}+\cdots+C_{n} t^{\alpha-n},
$$

where $C_{i} \in R, i=1,2, \ldots n$. 
Lemma 2.5. The relations

$$
I_{0+}^{\alpha} I_{0+}^{\beta} \varphi=I_{0+}^{\alpha+\beta} \varphi, D_{0+}^{\alpha} I_{0+}^{\alpha}=\varphi
$$

are valid in following case

$$
\operatorname{Re} \beta>0, \quad \operatorname{Re}(\alpha+\beta)>0, \quad \varphi \in L_{1}(0,1) .
$$

Lemma 2.6. Suppose that $h:(0,1) \rightarrow R$ satisfying that there exist constants $M>0, k<1$ and $\sigma \in(0, \alpha)$ such that

$$
|h(t)| \leq \frac{M}{t^{k}(1-t)^{\sigma}}, \quad t \in(0,1) .
$$

Then $u$ is a solution of

$$
\left\{\begin{array}{l}
D^{\alpha} u(t)+h(t)=0,0<t<1 \\
{\left.\left[I_{0^{+}}^{2-\alpha} u(t)\right]^{\prime}\right|_{t=0}=0} \\
u(1)=0
\end{array}\right.
$$

if and only if

$$
u(t)=\frac{1}{\Gamma(\alpha)}\left[\int_{0}^{t}\left(t^{\alpha-2}(1-s)^{\alpha-1}-(t-s)^{\alpha-1}\right) h(s) d s+t^{\alpha-2} \int_{t}^{1}(1-s)^{\alpha-1} h(s) d s\right] .
$$

Proof. We may apply Lemma 2.4 to reduce BVP(2.1) to an equivalent integral equation

$$
u(t)=-\int_{0}^{t} \frac{(t-s)^{\alpha-1}}{\Gamma(\alpha)} h(s) d s+c_{1} t^{\alpha-1}+c_{2} t^{\alpha-2}
$$

for some $c_{i} \in R, i=1,2$. By the assumption imposed on $h$, we know that

$$
\begin{aligned}
& \left|\int_{0}^{t} \frac{(t-s)^{\alpha-1}}{\Gamma(\alpha)} h(s) d s\right| \leq \int_{0}^{t} \frac{(t-s)^{\alpha-1}}{\Gamma(\alpha)}|h(s)| d s \\
\leq & \int_{0}^{t} \frac{(t-s)^{\alpha-1}}{\Gamma(\alpha)} \frac{M}{s^{k}(1-s)^{\sigma}} d s \leq M \int_{0}^{1} \frac{(1-s)^{\alpha-1}}{\Gamma(\alpha)} \frac{1}{s^{k}(1-s)^{\sigma}} d s \\
= & \frac{M}{\Gamma(\alpha)} \int_{0}^{1}(1-s)^{\alpha-\sigma-1} s^{-k} d s=\frac{M}{\Gamma(\alpha)} \mathbf{B}(\alpha-\sigma, 1-k) .
\end{aligned}
$$

So $u(t)$ is well defined and is continuous on $(0,1]$. Then We get

$$
t^{2-\alpha} u(t)=-t^{2-\alpha} \int_{0}^{t} \frac{(t-s)^{\alpha-1}}{\Gamma(\alpha)} h(s) d s+c_{1} t+c_{2}
$$

and

$$
\left[I_{0}^{2-\alpha} u(t)\right]^{\prime}=-\int_{0}^{t} h(s) d s+c_{1} \Gamma(\alpha) .
$$

Then $\left.\left[I_{0}^{2-\alpha} u(t)\right]^{\prime}\right|_{t=0}=0$ implies $c_{1}=0 . u(1)=0$ implies that

$$
c_{2}=\int_{0}^{1} \frac{(1-s)^{\alpha-1}}{\Gamma(\alpha)} h(s) d s .
$$

Therefore, the unique solution of $\operatorname{BVP}(2.1)$ is

$$
u(t)=-\int_{0}^{t} \frac{(t-s)^{\alpha-1}}{\Gamma(\alpha)} h(s) d s+\frac{t^{\alpha-2}}{\Gamma(\alpha)} \int_{0}^{1}(1-s)^{\alpha-1} h(s) d s .
$$

Then (2.2) holds. Reciprocally, let $u$ satisfy (2.2). Then

$$
\left.\left[I_{0^{+}}^{2-\alpha} u(t)\right]^{\prime}\right|_{t=0}=0, u(1)=0
$$

furthermore, we have $D^{\alpha} u(t)=-h(t)$. The proof is complete. 
Lemma 2.7. Suppose that $\beta \in(0,1)$ and $h:(0,1) \rightarrow[0, \infty)$ satisfying that there exist constants $M>0$, $k<1$ and $\sigma \in(0, \alpha)$ such that

$$
h(t) \leq \frac{M}{t^{k}(1-t)^{\sigma}}, \quad t \in(0,1) .
$$

If $u$ is the solution of $\operatorname{BVP}(2.1)$, then

$$
\inf _{t \in(0, \beta]} t^{2-\alpha} u(t) \geq\left(1-\beta^{2-\alpha}\right) \sup _{t \in(0,1]} t^{2-\alpha} u(t) .
$$

Proof. One sees from Lemma 2.6 that $u$ satisfies (2.2). Let

$$
G(t, s)=\frac{1}{\Gamma(\alpha)} \begin{cases}t^{\alpha-2}(1-s)^{\alpha-1}-(t-s)^{\alpha-1}, & 0 \leq s \leq t \leq 1, t \neq 0 \\ t^{\alpha-2}(1-s)^{\alpha-1}, & 0<t \leq s \leq 1\end{cases}
$$

It is easy to see that

$$
\Gamma(\alpha) t^{2-\alpha} G(t, s) \leq(1-s)^{\alpha-1} \text { for all } t, s \in(0,1] .
$$

On the other hand, we have

$$
\Gamma(\alpha) t^{2-\alpha} G(t, s) \geq\left(1-\beta^{2-\alpha}\right)(1-s)^{\alpha-1}, \quad t \in(0, \beta], s \in(0,1] .
$$

It follows from (2.2) that $u(t)=\int_{0}^{1} G(t, s) h(s) d s \geq 0$. Hence

$$
\begin{aligned}
\min _{t \in(0, \beta]} t^{2-\alpha} u(t) & =\int_{0}^{1} \min _{t \in(0, \beta]} t^{2-\alpha} G(t, s) h(s) d s \\
& \geq \int_{0}^{1}\left(1-\beta^{2-\alpha}\right) \frac{(1-s)^{\alpha-1}}{\Gamma(\alpha)} h(s) d s \\
& \geq\left(1-\beta^{2-\alpha}\right) \int_{0}^{1} t^{2-\alpha} G(t, s) h(s) d s .
\end{aligned}
$$

Hence

$$
\min _{t \in(0, \beta]} t^{2-\alpha} u(t) \geq\left(1-\beta^{2-\alpha}\right) \sup _{t \in(0,1]} t^{2-\alpha} u(t) .
$$

Then (2.3) holds. The proof is completed.

For our construction, we let

$$
X=\left\{x \in C(0,1]: \text { there exists the limit } \lim _{t \rightarrow 0} t^{2-\alpha} x(t)\right\}
$$

with the norm $\|u\|=\sup _{t \in(0,1]}\left|t^{2-\alpha} u(t)\right|$ for $u \in X$. Then $X$ is a Banach space. We seek solutions of $\operatorname{BVP}(1.1)$ that lie in the cone

$$
P=\left\{u \in X: u(t) \geq 0, t \in(0,1], \inf _{t \in(0, \beta]} t^{2-\alpha} u(t) \geq\left(1-\beta^{2-\alpha}\right)\|u\|\right\} .
$$

Define the operator $T$ on $P$ by

$$
(T u)(t)=\int_{0}^{1} G(t, s) f(s, u(s)) d s .
$$

Lemma 2.8. Suppose that $x \rightarrow f\left(t, t^{\alpha-2} x\right)$ is continuous on $(0,1) \times[0, \infty)$ and satisfies that

- for each $r>0$ there exist constants $M_{r}>0, k<1$ and $\sigma \in(0,1)$ such that

$$
f\left(t, t^{\alpha-2} x\right) \leq \frac{M_{r}}{t^{k}(1-t)^{\sigma}} \text { for all } t \in(0,1),|x| \leq r .
$$

Then $T: P \rightarrow P$ is well defined and is completely continuous. 
Proof. We divide the proof into four steps.

Step 1. We prove that $T: P \rightarrow P$ is well defined.

For $u \in P$, we find $u(t) \geq 0$ for all $t \in(0,1]$ and there exits $r>0$ such that

$$
\|u\|=\sup _{t \in(0,1]}\left|t^{2-\alpha} u(t)\right|<r .
$$

Then there exist constants $M_{r}>0, k<1$ and $\sigma \in(0, \alpha)$ such that

$$
0 \leq f(t, x(t))=f\left(t, t^{\alpha-2} t^{2-\alpha} x(t)\right) \leq \frac{M_{r}}{t^{k}(1-t)^{\sigma}}
$$

for all $t \in(0,1)$. Since $f$ is nonnegative, by $(2.2)$, we know that $(T u)(t) \geq 0$ for all $t \in(0,1]$. By Lemma 2.6 , we know that $T u \in C^{0}(0,1]$.

On the other hand, the definition of $T,(2.4)$ and (2.6) imply that

$$
\begin{aligned}
t^{2-\alpha}|(T u)(t)| & =\left|\int_{0}^{1} t^{2-\alpha} G(t, s) f(s, u(s)) d s\right| \\
& \leq \int_{0}^{1} \frac{(1-s)^{\alpha-1-\sigma}}{\Gamma(\alpha)}(1-s)^{\sigma} f\left(s, s^{\alpha-2} s^{2-\alpha} u(s)\right) d s \\
& \leq \int_{0}^{1} \frac{(1-s)^{\alpha-1-\sigma}}{\Gamma(\alpha)} M_{r} s^{-k} d s \\
& \leq \frac{M_{r}}{\Gamma(\alpha)} \mathbf{B}(\alpha-\sigma, 1-k) .
\end{aligned}
$$

By (2.2), we know that

$$
t^{2-\alpha} u(t)=\frac{t^{2-\alpha}}{\Gamma(\alpha)} \int_{0}^{t}\left(t^{\alpha-2}(1-s)^{\alpha-1}-(t-s)^{\alpha-1}\right) f(s, u(s)) d s+\frac{1}{\Gamma(\alpha)} \int_{t}^{1}(1-s)^{\alpha-1} f(s, u(s)) d s .
$$

It is easy to show that $\lim _{t \rightarrow 0} t^{2-\alpha}(T u)(t)=\frac{1}{\Gamma(\alpha)} \int_{0}^{1}(1-s)^{\alpha-1} f(s, u(s)) d s$. By the method used in Lemma 2.7 (replace $h$ by $f$ ), we get

$$
\inf _{t \in(0, \beta]} t^{2-\alpha}(T u)(t) \geq\left(1-\beta^{2-\alpha}\right) \sup _{t \in(0,1]} t^{2-\alpha}(T u)(t) .
$$

So $T u \in P$. So $T: P \rightarrow P$ is well defined.

Step 2. $T$ is continuous.

Let $\left\{y_{n}\right\}$ be a sequence such that $y_{n} \rightarrow y$ in $P$. Let

$$
\max \left\{\sup _{t \in(0,1]} t^{2-\alpha}\left|y_{n}(t)\right|, \sup _{t \in(0,1]} t^{2-\alpha}|y(t)|\right\} \leq r .
$$

Then there exist constants $M_{r}>0, k<1$ and $\sigma \in(0, \alpha)$ such that $f(t, x(t))=f\left(t, t^{\alpha-2} t^{2-\alpha} x(t)\right) \leq \frac{M}{t^{k}(1-t)^{\sigma}}$ for all $t \in(0,1)$. Then for $t \in(0,1]$, we have

$$
\begin{aligned}
t^{2-\alpha}\left|\left(T y_{n}\right)(t)-(T y)(t)\right| & =\left|\int_{0}^{1} t^{2-\alpha} G(t, s) f\left(s, y_{n}(s)\right) d s-\int_{0}^{1} t^{2-\alpha} G(t, s) f(s, y(s)) d s\right| \\
& \leq \int_{0}^{1} t^{2-\alpha} G(t, s)\left|f\left(s, y_{n}(s)\right)-f(s, y(s))\right| d s \\
& \leq 2 \frac{1}{\Gamma(\alpha)} \int_{0}^{1}(1-s)^{\alpha-1} \frac{M_{r}}{s^{k}(1-s)^{\sigma}} d s \\
& \leq 2 \frac{M_{r}}{\Gamma(\alpha)} \mathbf{B}(\alpha-\sigma, 1-k) .
\end{aligned}
$$


Since

$$
f\left(t, t^{\alpha-2} x\right)
$$

is continuous in $x$, we have

$$
\left\|T y_{n}-T y\right\| \rightarrow 0
$$

as $n \rightarrow \infty$.

Step 3. $T$ maps bounded sets into bounded sets in $P$.

It suffices to show that for each $l>0$, there exists a positive number $L>0$ such that for each

$$
x \in M=\{y \in P:\|y\| \leq l\}
$$

, we have

$$
\|T y\| \leq L
$$

For $y \in M$, we have

$$
\sup _{t \in(0,1]} t^{2-\alpha}|y(t)| \leq l \text {. }
$$

Then there exist constants $M_{r}>0, k<1$ and $\sigma \in(0, \alpha)$ such that $f(t, y(t))=f\left(t, t^{\alpha-2} t^{2-\alpha} y(t)\right) \leq \frac{M_{l}}{t^{k}(1-t)^{\sigma}}$ for all $t \in(0,1)$.

By the definition of $T$, we get

$$
\begin{aligned}
t^{2-\alpha}|(T y)(t)| & =\int_{0}^{1} t^{2-\alpha} G(t, s) f(s, y(s)) d s \\
& \leq \frac{1}{\Gamma(\alpha)} \int_{0}^{1}(1-s)^{\alpha-1} f(s, y(s)) d s \\
& \leq \frac{1}{\Gamma(\alpha)} \int_{0}^{1}(1-s)^{\alpha-1} \frac{M_{l}}{s^{k}(1-s)^{\sigma}} d s \\
& =\frac{M_{l}}{\Gamma(\alpha)} \mathbf{B}(\alpha-\sigma, 1-k) .
\end{aligned}
$$

It follows that

$$
\|T y\| \leq \frac{M_{l}}{\Gamma(\alpha)} \mathbf{B}(\alpha-\sigma, 1-k) \text { for each } y \in\{y \in P:\|y\| \leq l\} .
$$

So $T$ maps bounded sets into bounded sets in $P$.

Step 4. $T$ maps bounded sets into pre-compact sets.

Let $M=\{y \in P:\|y\| \leq l\}$ be a bounded set in $X$. We prove that $\left\{t^{2-\alpha} T u(t): u \in M\right\}$ is equicontinuous on compact sub interval of $(0,1]$ and is equi-convergent at $t=0$.

Firstly, let $t_{1}, t_{2} \in[a, b] \subset(0,1]$ with $0<a<b \leq 1, t_{1}<t_{2}$ and

$$
y \in M=\{y \in P:\|y\| \leq l\}
$$

defined in Step 3. For $y \in M$, we have

$$
\sup _{t \in(0,1]} t^{2-\alpha}|y(t)| \leq l \text {. }
$$

Then there exist constants $M_{r}>0, k<1$ and $\sigma \in(0, \alpha)$ such that

$$
f(t, y(t))=f\left(t, t^{\alpha-2} t^{2-\alpha} y(t)\right) \leq \frac{M_{l}}{t^{k}(1-t)^{\sigma}}
$$

for all $t \in(0,1)$. By the definition of $T$, we have

$$
(T y)(t)=-\int_{0}^{t} \frac{(t-s)^{\alpha-1}}{\Gamma(\alpha)} f(s, y(s)) d s+\frac{t^{\alpha-2}}{\Gamma(\alpha)} \int_{0}^{1}(1-s)^{\alpha-1} f(s, y(s)) d s .
$$


We have

$$
\begin{aligned}
& \Gamma(\alpha)\left|t_{1}^{2-\alpha}(T y)\left(t_{1}\right)-t_{2}^{2-\alpha}(T y)\left(t_{2}\right)\right| \\
& =\left|t_{2}^{2-\alpha} \int_{0}^{t_{2}}\left(t_{2}-s\right)^{\alpha-1} f(s, y(s)) d s-t_{1}^{2-\alpha} \int_{0}^{t_{1}}\left(t_{1}-s\right)^{\alpha-1} f(s, y(s)) d s\right| \\
& \leq\left|t_{1}^{2-\alpha}-t_{2}^{2-\alpha}\right| \int_{0}^{t_{2}}\left(t_{2}-s\right)^{\alpha-1}|f(s, y(s))| d s \\
& +t_{1}^{2-\alpha} \int_{t_{1}}^{t_{2}}\left(t_{2}-s\right)^{\alpha-1}\left|f\left(s, s^{\alpha-2} s^{2-\alpha} y(s)\right)\right| d s \\
& +t_{1}^{2-\alpha} \int_{0}^{t_{1}}\left[\left|\left(t_{2}-s\right)^{\alpha-1}-\left(t_{1}-s\right)^{\alpha-1}\right|\right]\left|f\left(s, s^{\alpha-2} s^{2-\alpha} y(s)\right)\right| d s \\
& \leq\left|t_{1}^{2-\alpha}-t_{2}^{2-\alpha}\right| \int_{0}^{1}(1-s)^{\alpha-1} \frac{M_{l}}{s^{k}(1-s)^{\sigma}} d s \\
& +\int_{t_{1}}^{t_{2}}(1-s)^{\alpha-1} \frac{M_{l}}{s^{k}(1-s)^{\sigma}} d s \\
& +\int_{0}^{t_{1}}\left[\left(t_{2}-s\right)^{\alpha-1}-\left(t_{1}-s\right)^{\alpha-1}\right] \frac{M_{l}}{s^{k}(1-s)^{\sigma}} d s \\
& \leq\left|t_{1}^{2-\alpha}-t_{2}^{2-\alpha}\right| M_{l} \mathbf{B}(\alpha-\sigma, 1-k)+M_{l} \int_{t_{1}}^{t_{2}}(1-s)^{\alpha-\sigma-1} s^{-k} d s \\
& +M_{l} \int_{0}^{t_{1}}\left(t_{2}-t_{1}\right)^{\alpha-1} \frac{1}{s^{k}(1-s)^{\sigma}} d s \\
& \leq\left|t_{1}^{2-\alpha}-t_{2}^{2-\alpha}\right| M_{l} \mathbf{B}(\alpha-\sigma, 1-k)+M_{l} \int_{t_{1}}^{t_{2}}(1-s)^{\alpha-\sigma-1} s^{-k} d s \\
& +M_{l}\left(t_{2}-t_{1}\right)^{\alpha-1} \int_{0}^{t_{1}} \frac{1}{s^{k}\left(t_{1}-s\right)^{\sigma}} d s \\
& =\left|t_{1}^{2-\alpha}-t_{2}^{2-\alpha}\right| M_{l} \mathbf{B}(\alpha-\sigma, 1-k)+M_{l} \int_{t_{1}}^{t_{2}}(1-s)^{\alpha-\sigma-1} s^{-k} d s \\
& +M_{l}\left(t_{2}-t_{1}\right)^{\alpha-1} t_{1}^{1-\sigma-k} \int_{0}^{1}(1-w)^{-\sigma} w^{-k} d w \\
& \leq\left|t_{1}^{2-\alpha}-t_{2}^{2-\alpha}\right| M_{l} \mathbf{B}(\alpha-\sigma, 1-k)+M_{l} \int_{t_{1}}^{t_{2}}(1-s)^{\alpha-\sigma-1} s^{-k} d s \\
& +M_{l}\left(t_{2}-t_{1}\right)^{\alpha-1} \max \left\{a^{1-\sigma-k}, b^{1-\sigma-k}\right\} \mathbf{B}(1-\sigma, 1-k) .
\end{aligned}
$$

As $t_{1} \rightarrow t_{2}$, the right-hand side of the above inequality tends to zero uniformly. So $\left\{t^{2-\alpha} T u(t): u \in M\right\}$ is equi-continuous on $[a, b]$.

Secondly, we have

$$
\begin{aligned}
& \left|t^{2-\alpha}(T y)(t)-\frac{1}{\Gamma(\alpha)} \int_{0}^{1}(1-s)^{\alpha-1} f(s, y(s)) d s\right| \\
\leq & t^{2-\alpha} \int_{0}^{t} \frac{(t-s)^{\alpha-1}}{\Gamma(\alpha)}\left|f\left(s, s^{\alpha-2} s^{2-\alpha} y(s)\right)\right| d s \\
\leq & t^{2-\alpha} \int_{0}^{1} \frac{(1-s)^{\alpha-1}}{\Gamma(\alpha)} \frac{M_{l}}{s^{k}(1-s)^{\sigma}} d s=t^{2-\alpha} M_{l} \frac{\mathbf{B}(\alpha-\sigma, 1-k)}{\Gamma(\alpha)} .
\end{aligned}
$$

The right-hand side of the above inequality tends to zero uniformly as $t \rightarrow 0$. Then $\left\{t^{2-\alpha} T u(t): u \in M\right\}$ is equi-convergent at $t=0$. 
Therefore, $T M$ is compact. From above discussion, $T$ is completely continuous. The proof is complete.

\section{Main Results}

In this section, we prove the main results. Let $M=\frac{\mathbf{B}(\alpha-\sigma, 1-k)}{\Gamma(\alpha)}, W=\frac{1-\beta^{2-\alpha}}{\Gamma(\alpha)} \int_{0}^{\beta}(1-s)^{\alpha-\sigma-1} s^{-k} d s$.

Theorem 3.1. Suppose that $f:(0,1) \times[0, \infty) \rightarrow[0, \infty)$ is continuous, $f(t, 0) \not \equiv 0$ on $(0,1)$ and satisfies

- there exist constants $k<1, \sigma \in(0,1), e_{1}, e_{2}$ and $c$ such that

$$
0<e_{1}<e_{2}<\frac{e_{2}}{1-\beta^{2-\alpha}}<c, W c>M e_{2},
$$

and

(D1) $f\left(t, t^{\alpha-2} u\right) \leq \frac{c}{M} \frac{1}{t^{k}(1-t)^{\sigma}}$ for $t \in(0,1), u \in[0, c]$;

(D2) $f\left(t, t^{\alpha-2} u\right) \leq \frac{e_{1}}{M} \frac{1}{t^{k}(1-t)^{\sigma}}$ for $t \in(0,1)$ and $u \in\left[0, e_{1}\right]$;

(D3) $f\left(t, t^{\alpha-2} u\right) \geq \frac{e_{2}}{W} \frac{1}{t^{k}(1-t)^{\sigma}}$ for $t \in(0, \beta]$ and $u \in\left[e_{2}, \frac{e_{2}}{\beta}\right]$.

Then $\operatorname{BVP}(1.1)$ has at least three unbounded positive solutions $x_{1}, x_{2}$ and $x_{3}$ satisfying

$$
\sup _{t \in(0,1]} t^{2-\alpha} x_{1}(t)<e_{1}, \inf _{t \in(0, \beta]} t^{2-\alpha} x_{2}(t)>e_{2}
$$

and

$$
\sup _{t \in(0,1]} t^{2-\alpha} x_{3}(t)>e_{1}, \inf _{t \in(0, \beta]} t^{2-\alpha} x_{3}(t)<e_{2} .
$$

Proof. Define the functional $\psi$ by

$$
\psi(x)=\inf _{t \in(0, \beta]} t^{2-\alpha} x(t) \text { for } x \in P .
$$

It is easy to see that $\psi$ is a nonnegative convex continuous functional on the cone $P . \psi(y) \leq\|y\|$ for all $y \in P$. For $x \in P$, it follows from Lemma 2.8 that $T P \subseteq P$ and $T: P \rightarrow P$ is completely continuous.

Corresponding to Lemma 2.1, choose

$$
d=\frac{e_{2}}{1-\beta^{2-\alpha}}, \quad b=e_{2}, \quad a=e_{1} .
$$

Then $0<a<b<d<c$. We divide the remainder of the proof into five steps.

Step 1. Prove that $T\left(\overline{P_{c}}\right) \subset \overline{P_{c}}$.

For $x \in \overline{P_{c}}$, one has $\|x\| \leq c$. Then

$$
0 \leq t^{2-\alpha} x(t) \leq c, t \in(0,1] .
$$

It follows from (D1) that

$$
f(t, x(t))=f\left(t, t^{\alpha-2} t^{2-\alpha} x(t)\right) \leq \frac{c}{M} \frac{1}{t^{k}(1-t)^{\sigma}}, t \in(0,1) .
$$

Then $T x \in P$ implies that

$$
\begin{aligned}
\|T x\| & =\sup _{t \in(0,1]} t^{2-\alpha}(T x)(t) \\
& =\sup _{t \in(0,1]} \int_{0}^{1} t^{2-\alpha} G(t, s) f(s, x(s)) d s \\
& \leq \sup _{t \in(0,1]} \int_{0}^{1} t^{2-\alpha} G(t, s) \frac{c}{M} \frac{1}{s^{k}(1-s)^{\sigma}} d s \\
& \leq \frac{1}{\Gamma(\alpha)} \frac{c}{M} \int_{0}^{1}(1-s)^{\alpha-1} \frac{1}{s^{k}(1-s)^{\sigma}} d s \\
& =\frac{1}{\Gamma(\alpha)} \frac{c}{M} \mathbf{B}(\alpha-\sigma-1,1-k)=c .
\end{aligned}
$$


Then $T x \in \overline{P_{c}}$, Hence $T\left(\overline{P_{c}}\right)$. This completes the proof of Step 1.

Step 2. Prove that

$$
\{y \in P(\psi ; b, d) \mid \psi(y)>b\}=\left\{y \in P\left(\psi ; e_{2}, \frac{e_{2}}{1-\beta^{2-\alpha}}\right): \psi(y)>e_{2}\right\} \neq \emptyset
$$

and $\psi(T y)>b=e_{2}$ for $y \in P\left(\psi ; e_{2}, \frac{e_{2}}{1-\beta^{2-\alpha}}\right)$.

It is easy to see that $\left\{x \in P\left(\psi ; e_{2}, \frac{e_{2}}{1-\beta^{2-\alpha}}\right), \psi(x)>e_{2}\right\} \neq \emptyset$. For $x \in P\left(\psi ; e_{2}, \frac{e_{2}}{1-\beta^{2-\alpha}}\right)$, then $\psi(x) \geq e_{2}$ and $\|x\| \leq \frac{e_{2}}{1-\beta^{2-\alpha}}$. Then

$$
\inf _{t \in(0, \beta]} t^{2-\alpha} x(t) \geq e_{2}, \sup _{t \in(0,1]} t^{2-\alpha} x(t) \leq \frac{e_{2}}{1-\beta^{2-\alpha}}
$$

Hence

$$
e_{2} \leq t^{2-\alpha} x(t) \leq \frac{e_{2}}{1-\beta^{2-\alpha}}, t \in(0, \beta]
$$

Hence (D3) implies that

$$
f(t, x(t)) \geq \frac{e_{2}}{W} \frac{1}{t^{k}(1-t)^{\sigma}}, t \in(0, \beta] .
$$

Since $T y \in P$. We get

$$
\begin{aligned}
\psi(T x) & =\inf _{t \in(0, \beta]} \int_{0}^{1} t^{2-\alpha} G(t, s) f(s, x(s)) d s \\
& >\inf _{t \in(0, \beta]} \int_{0}^{\beta} t^{2-\alpha} G(t, s) f(s, x(s)) d s \\
& \geq \int_{0}^{\beta}\left(1-\beta^{2-\alpha}\right) \frac{(1-s)^{\alpha-1}}{\Gamma(\alpha)} f(s, x(s)) d s \\
& \geq\left(1-\beta^{2-\alpha}\right) \int_{0}^{\beta} \frac{(1-s)^{\alpha-1}}{\Gamma(\alpha)} \frac{e_{2}}{W} \frac{1}{s^{k}(1-s)^{\sigma}} d s \\
& =e_{2} .
\end{aligned}
$$

This completes the proof of Step 2 .

Step 3. Prove that $\|T y\|<a=e_{1}$ for $y \in P$ with $\|y\| \leq a$.

For $x \in \overline{P_{e_{1}}}$, we have

$$
\sup _{t \in(0,1]} t^{2-\alpha} x(t) \leq e_{1}=a
$$

It follows from (D2) and $T x \in P$ that

$$
f(t, x(t)) \leq \frac{e_{1}}{M} \frac{1}{t^{k}(1-t)^{\sigma}}, t \in(0,1] .
$$

The proof is similar to that of Step 1. Then $\|T y\|<e_{1}$ for $\|y\| \leq e_{1}$. This completes that proof of Step 3 .

Step 4. Prove that $\psi(T y)>b$ for $y \in P(\psi ; b, c)$ with $\|T y\|>d$.

For $x \in P(\psi ; b, c)=P\left(\psi, e_{2}, c\right)$ and $\|T x\|>d=\frac{e_{2}}{1-\beta^{2-\alpha}}$, then

$$
\inf _{t \in(0, \beta]} t^{2-\alpha} x(t) \geq e_{2}, \sup _{t \in(0,1]} t^{2-\alpha}(T x)(t) \geq \frac{e_{2}}{1-\beta^{2-\alpha}} \text { and }\|x\|=\sup _{t \in(0,1]} t^{2-\alpha} x(t) \leq c .
$$


Hence we have from $T x \in P$ that

$$
\begin{aligned}
\psi(T x) & =\inf _{t \in(0, \beta]} t^{2-\alpha}(T x)(t) \\
& =\left(1-\beta^{2-\alpha}\right) \sup _{t \in(0,1]}(T x)(t) \\
& \geq\left(1-\beta^{2-\alpha}\right) \frac{e_{2}}{1-\beta^{2-\alpha}} \\
& =b .
\end{aligned}
$$

This completes the proof of Step 4 .

From above steps, (E1), (E2) and (E3) of Lemma 2.1 are satisfied. Then, by Lemma 2.1, $T$ has three fixed points $x_{1}, x_{2}$ and $x_{3} \in \overline{P_{c}}$ such that

$$
\left\|x_{1}\right\|<a, \psi_{1}\left(x_{2}\right)>b,\left\|x_{3}\right\| \geq a, \psi_{1}\left(x_{3}\right) \leq b,
$$

i.e., $x_{1}, x_{2}$ and $x_{3}$ satisfy $(3.1)$ and (3.2).

Finally, we prove that $x_{i}(i=1,2,3)$ are unbounded. In fact, $f(t, 0) \not \equiv 0$ on $(0,1)$ implies that $x_{i}(t) \not \equiv 0$. We have

$$
\inf _{t \in(0, \beta]} t^{2-\alpha} x_{i}(t) \geq\left(1-\beta^{2-\beta}\right) \sup _{t \in(0,1]} t^{2-\alpha} x_{i}(t)
$$

So

$$
x_{i}(t) \geq t^{\alpha-2}\left(1-\beta^{2-\beta}\right) \sup _{t \in(0,1]} t^{2-\alpha} x_{i}(t), \quad t \in(0, \beta] .
$$

Then $\lim _{t \rightarrow 0} x_{i}(t)=\infty$. So $x_{i}$ is bounded. Hence $\operatorname{BVP}(1.1)$ has at least three unbounded positive solutions. The proof is complete.

Theorem 3.2. Suppose that $f:(0,1) \times[0, \infty) \rightarrow[0, \infty)$ is continuous, $f(t, 0) \not \equiv 0$ on $(0,1)$ and satisfies

- there exist positive numbers $k<1, \sigma \in(0,1), a<b<c$ such that $W b>M a$, and

(E1) $f\left(t, t^{\alpha-2} u\right) \geq \frac{c}{W} \frac{1}{t^{k}(1-t)^{\sigma}}$ for $t \in(0, \beta], u \in\left[c, c /\left(1-\beta^{2-\alpha}\right)\right]$;

(E2) $f\left(t, t^{\alpha-2} u\right) \leq \frac{b}{M} \frac{1}{t^{k}(1-t)^{\sigma}}$ for $t \in(0,1)$ and $u \in[0, b]$;

(E3) $f\left(t, t^{\alpha-2} u\right) \geq \frac{a}{W} \frac{1}{t^{k}(1-t)^{\sigma}}$ for $t \in[\beta, 1)$ and $u \in\left[\left(1-\beta^{2-\alpha}\right) a, a\right]$.

Then BVP(1.1) has at least two unbounded positive solutions $x_{1}$ and $x_{2}$ satisfying

$$
\sup _{t \in(0,1]} t^{2-\alpha} x_{1}(t)>a, \sup _{t \in(0,1]} t^{2-\alpha} x_{1}(t)<b,
$$

and

$$
\sup _{t \in(0,1]} t^{2-\alpha} x_{2}(t)>b, \inf _{t \in(0, \beta]} t^{2-\alpha} x_{2}(t)<c .
$$

Proof. Define the nonnegative, increasing and continuous functionals $\gamma, \theta, \alpha: P \rightarrow I$ by

$$
\begin{aligned}
& \gamma(x)=\inf _{t \in(0, \beta]} t^{2-\alpha} x(t), \quad x \in P, \\
& \theta(x)=\sup _{t \in(0,1]} t^{2-\alpha} x(t), \quad x \in P, \\
& \alpha(x)=\sup _{t \in(0,1]} t^{2-\alpha} x(t), \quad x \in P .
\end{aligned}
$$

It is easy to see that $\theta(0)=0$ and

$$
\gamma(x) \leq \theta(x) \leq \alpha(x), \quad x \in P
$$

and for $x \in P$ we have $\gamma(x) \geq\left(1-\beta^{2-\alpha}\right)\|x\|, \theta(\nu x) \leq \nu \theta(x)$ for all $\nu \in[0,1]$ and $x \in P$. From Lemma 2.8, we have $T P \subset P$ and $T$ is completely continuous. Hence (i)-(iii) in Lemma 2.2 hold. 
To obtain two positive solutions of BVP(1.1), it suffices to show that the condition (iv) in Lemma 2.2 holds.

First, we verify that

$$
\gamma(T x)>c \text { for alll } x \in \partial P(\gamma, c) .
$$

Since $x \in \partial P(\gamma, c)$, we get $\inf _{t \in(0, \beta]} t^{2-\alpha} x(t)=c$. Then $\|x\| \leq \frac{1}{1-\beta^{2-\alpha}} \gamma(x) \leq \frac{c}{1-\beta^{2-\alpha}}$. Then

$$
c \leq t^{2-\alpha} x(t) \leq \frac{c}{1-\beta^{2-\alpha}} \text { for all } t \in(0, \beta] .
$$

Hence (E1) implies

$$
f(t, x(t)) \geq \frac{c}{W} \frac{1}{t^{k}(1-t)^{\sigma}}, \quad t \in(0, \beta]
$$

So we get from $T x \in P$ that

$$
\begin{aligned}
\gamma(T x)(t) & =\inf _{t \in(0, \beta]} t^{2-\alpha} \int_{0}^{1} G(t, s) f(s, x(s)) d s \\
& >\int_{0}^{\beta}\left(1-\beta^{2-\alpha}\right) \frac{(1-s)^{\alpha-1}}{\Gamma(\alpha)} f(s, x(s)) d s \\
& \geq\left(1-\beta^{2-\alpha}\right) \int_{0}^{\beta} \frac{(1-s)^{\alpha-1}}{\Gamma(\alpha)} \frac{c}{W} \frac{1}{s^{k}(1-s)^{\sigma}} d s \\
& \geq c .
\end{aligned}
$$

Secondly, we prove that

$$
\theta(T x)<b \text { for all } x \in \partial P(\theta, b) .
$$

Since $\theta(x)=b$, we get $\sup _{t \in(0,1]} t^{2-\alpha} x(t)=b$. Then

$$
t^{2-\alpha} x(t) \leq b \text { for all } t \in(0,1]
$$

Hence (E2) implies

$$
f(t, x(t)) \leq \frac{b}{M} \frac{1}{t^{k}(1-t)^{\sigma}}, t \in(0,1)
$$

So the definition of $T$ imply

$$
\begin{aligned}
\theta(T x) & =\sup _{t \in(0,1]} t^{2-\alpha}(T x)(t) \\
& \leq \sup _{t \in(0,1]} \int_{0}^{1} t^{2-\alpha} G(t, s) f(s, x(s)) d s \\
& \leq \frac{1}{\Gamma(\alpha)} \frac{b}{M} \int_{0}^{1}(1-s)^{\alpha-1} \frac{1}{s^{k}(1-s)^{\sigma}} d s \\
& =b .
\end{aligned}
$$

Finally, we prove that

$$
P(\alpha, a) \neq \emptyset, \quad \alpha(T x)>a \text { for all } x \in \partial P(\alpha, a) .
$$

It is easy to see that $P(\alpha, a) \neq \emptyset$. For $x \in \partial P(\alpha, a)$, we have $\sup _{t \in(0,1]} t^{2-\alpha} x(t)=a$. Then

$$
\left(1-\beta^{2-\alpha}\right) a \leq t^{2-\alpha} x(t) \leq a \text { for all } t \in(0, \eta] .
$$

Then (E3) implies

$$
f(t, x(t)) \geq \frac{a}{W} \frac{1}{t^{k}(1-t)^{\sigma}}, t \in(0, \eta) .
$$

Similarly to the first step, we can prove that $\alpha(T x)>a$. It follows from above discussion that all conditions in Lemma 2.2 are satisfied. Then $T$ has two fixed points $x_{1}, x_{2}$ in $P$. It is easy to show that $x_{i}$ is unbounded. So BVP(1.1) has two positive solutions $x_{1}$ and $x_{2}$ satisfying (3.3) and (3.4). The proof is complete. 
Theorem 3.3. Suppose that $f:(0,1) \times[0, \infty) \rightarrow[0, \infty)$ is continuous, $f(t, 0) \not \equiv 0$ on $(0,1)$ and satisfies

- there exist positive numbers $k<1, \sigma \in(0,1), a<\beta^{\alpha} b<b<c$ such that $W c>M b$, and

(E4) $f\left(t, t^{\alpha-2} u\right) \leq \frac{c}{M} \frac{1}{t^{k}(1-t)^{\sigma}}$ for $t \in(0,1), u \in\left[0, c /\left(1-\beta^{2-\alpha}\right)\right]$;

(E5) $f\left(t, t^{\alpha-2} u\right) \geq \frac{b}{W} \frac{1}{t^{k}(1-t)^{\sigma}}$ for $t \in(0, \beta]$ and $u \in\left[\left(1-\beta^{2-\alpha}\right) b, b\right]$;

(E6) $f\left(t, t^{\alpha-2} u\right) \leq \frac{a}{M} \frac{1}{t^{k}(1-t)^{\sigma}}$ for $t \in(0,1)$ and $u \in[0, a]$.

Then BVP(1.1) has at least two unbounded positive solutions $x_{1}$ and $x_{2}$ satisfying (3.3) and (3.4).

Proof. Let the nonnegative, increasing and continuous functionals $\gamma, \theta, \alpha: P \rightarrow I$ be defined in the proof of Theorem 3.2. By using Lemma 2.3, the remainder of the proof is similar to that of the proof of Theorem 3.2 and is omitted.

\section{References}

[1] K. S. Miller, B. Ross, An Introduction to the Fractional Calculus and Fractional Differential Equation, Wiley, New York, 1993.

[2] S. G. Samko, A. A. Kilbas, O. I. Marichev, Fractional Integral and Derivative. Theory and Applications, Gordon and Breach, 1993.

[3] Z. Bai, H. Lv, Positive solutions for boundary value problems of nonlinear fractional differential equations, J. Math. Anal. Appl. 311 (2005), 495-505.

[4] A. A. Kilbas and J. J. Trujillo, Differential equations of fractional order: methods, results and problems-I, Applicable Analysis, 78 (2001), 153-192.

[5] A. Arara, M. Benchohra, N. Hamidi, and J. J. Nieto, Fractional order differential equations on an unbounded domain, Nonlinear Analysis TMA, 72 (2010), 580-586,

[6] Z. Bai, On positive solutions of a nonlocal fractional boundary value problem, Nonlinear Analysis, 72 (2010), 916-924.

[7] R. Dehghant, K. Ghanbari, Triple positive solutions for boundary value problem of a nonlinear fractional differential equation, Bulletin of the Iranian Mathematical Society, 33 (2007), 1-14.

[8] S. Z. Rida, H. M. El-Sherbiny, and A. A. M. Arafa, On the solution of the fractional nonlinear Schrodinger equation, Physics Letters A, 372 (2008), 553-558.

[9] X. Xu, D. Jiang and C. Yuan, Multiple positive solutions for the boundary value problem of a nonlinear fractional differential equation, Nonlinear Analysis TMA, 71 (2009), 4676-4688.

[10] F. Zhang, Existence results of positive solutions to boundary value problem for fractional differential equation, Positivity, 13 (2008), 583-599.

[11] R. W. Leggett and L. R. Williams, Multiple positive fixed points of nonlinear operators on ordered Banach spaces, Indiana University Mathematics Journal 28 (1979), 673-688. 